\title{
Anaemia in Pregnancy - Not Just Iron Deficiency
}

\author{
Sant-Rayn Pasricha \\ Nossal Institute for Global Health, Faculty of Medicine, Dentistry and Health Sciences, The University of Melbourne, \\ Carlton, Vic., Australia
}

Anaemia remains an important global health problem. Almost $42 \%$ of all pregnant women worldwide (56 million) are thought to be anaemic. The prevalence is highest in low-income countries, especially in South and South East Asia, and Sub-Saharan Africa [1]. Iron deficiency is often considered the chief cause, and often deemed synonymous with anaemia in the public health context. For example, WHO guidelines assume that at least half the worldwide burden of anaemia is attributable to iron deficiency [2]. Indeed, the most recent global burden of disease report conflates iron deficiency with anaemia, using haemoglobin measurements to define the burden of disease attributable to iron deficiency [3].

However, beyond iron deficiency, anaemia has many possible causes. In the public health context, inherited red cell disorders, particularly thalassaemia and haemoglobinopathy (e.g. HbE) carrier and compound heterozygous and homozygous states, infections (especially malaria) and inflammation, and other micronutrient deficiencies (i.e. deficiencies of folate and vitamin B12 and A), may be important depending on the geographic setting and the population. Distinguishing these causes requires clinical observation and expensive laboratory investigation and, thus, relatively few studies have undertaken detailed analysis of the determinants of anaemia in populations. However, it is interesting that, where these have been performed, iron deficiency is sometimes a surprisingly minor player.

\section{KARGER}

E-Mail karger@karger.com

www.karger.com/aha
Approximately one third of pregnant women in Vietnam have been previously estimated to be anaemic [1]. In this issue of Acta Haematologica, Siridamrongvattana et al. [4] report in a cross-sectional study of 399 women in the first trimester of pregnancy in central Vietnam that they found an unexpectedly low prevalence of anaemia (19.3\%), iron deficiency (20.1\%) and iron-deficiency anaemia (6\%). Interestingly, the authors also found that most of the burden of anaemia could not be explained by iron deficiency: $31 \%$ of anaemic women had iron deficiency (ferritin $<15 \mu \mathrm{g} / \mathrm{l}$ ), $26 \%$ were found to have a thalassaemia mutation, and the remaining $43 \%$ of anaemic women had apparently replete iron stores and no evidence of haemoglobinopathy. There were no cases of severe anaemia. Inflammation did not appear to play a role. Other haematinic micronutrients (i.e. vitamins B12 and A, folate) were not measured.

These findings raise several interesting and important questions. Firstly, what is causing the unexplained anaemia? Other haematinic micronutrient deficiencies may account for some of these cases. Another possibility is that the authors' definition of iron deficiency is too specific, and that anaemic women with higher ferritin concentrations still have depleted iron stores. A study in nonpregnant women of reproductive age in Vietnam indicated that $77 \%$ of women who achieved an appropriate haemoglobin response to iron supplementation had a ferritin level $<30 \mu \mathrm{g} / \mathrm{l}$ [5], suggesting a higher cut-off for fer-

Dr. Sant-Rayn Pasricha

Nossal Institute for Global Health, Faculty of Medicine, Dentistry and Health Sciences The University of Melbourne

Level 4, 161 Barry Street, Carlton, VIC 3010 (Australia)

E-Mail sant-rayn.pasricha@ unimelb.edu.au 
ritin may be appropriate. Other useful indicators of iron status in pregnancy, such as the soluble transferrin receptor (and its ratio with ferritin), were not measured in this study. Thus, some of the unexplained anaemia may still be attributable to iron deficiency.

However, it would seem that a considerable burden of anaemia among pregnant women in this population is not due to iron deficiency. Although the authors conclude that iron deficiency is an important determinant of anaemia in this population, their data indicate that irondeficiency anaemia is in fact relatively uncommon. Furthermore, the data seem to indicate that the majority of iron-deficient women are not anaemic, and the majority of anaemic women are not iron deficient. The World Health Organization has recommended that pregnant women should be screened for anaemia, and that nonanaemic women be given iron/folic acid weekly, with anaemic women offered iron daily for the duration of pregnancy [2]. However, anaemia is likely to be a dismal test for iron status in this population, and providing daily iron based on anaemia status would miss non-anaemic iron-deficient women, and treat anaemic and non-irondeficient women in equal measure. The authors found that the contribution of haemoglobinopathies to the burden of anaemia was similar to that of iron deficiency. Inherited disorders of haemoglobin are highly prevalent in South East Asia, with an estimated $45 \%$ of the population carrying a 'significant' variant or alpha+ thalassaemia deletions [6]. Carriers of thalassaemia are unlikely to benefit from iron, as shown in the present study by median ferritin levels of $45 \mu \mathrm{g} / \mathrm{l}$ in this group. The safety of iron supplementation to pregnant women who carry haemoglobinopathies has not been widely studied, although some limited data suggest iron overload is unlikely to occur [7]; further studies are required to address this issue. However, anaemia-based stratification of iron supplementation in pregnancy may be problematic in South East Asia as it may not appropriately identify iron-deficient women.

The clinical implications of anaemia when not clearly due to iron deficiency remain uncertain. Although the effects of iron-deficiency anaemia on maternal and infant outcomes, and the effects of iron supplementation on improving these outcomes, have been studied and reviewed, little data exist for the maternal and infant effects of noniron-deficiency anaemia. Iron supplementation may not benefit, and could potentially harm (as shown for preschool children in malaria-endemic settings), pregnant women in settings where anaemia is due to conditions other than iron deficiency. The continued practice of attributing $50 \%$ of cases of anaemia to iron deficiency, and of using anaemia to indicate the prevalence of iron deficiency, may need to be reviewed. As performed by Siridamrongvattana et al. [4], a concerted effort to map and distinguish the epidemiology of anaemia from the epidemiology of haemoglobinopathy, malaria and iron deficiency in different low-income settings is needed.

\section{References}

1 Benoist B, McLean E, Egli I, Cogswell M: Worldwide prevalence of anaemia 1993-2005. Geneva, World Health Organization, 2008.

2 WHO: Guideline: intermittent iron and folic acid supplementation in non-anaemic pregnant women. Geneva, World Health Organization, 2012.

$>3$ Lim SS, Vos T, Flaxman AD, Danaei G, Shibuya K, Adair-Rohani H, et al: A comparative risk assessment of burden of disease and injury attributable to 67 risk factors and risk factor clusters in 21 regions, 1990-2010: a sys- tematic analysis for the Global Burden of Disease Study 2010. Lancet 2012;380:2224-2260.

4 Siridamrongvattana S, Hoa NV, Sanchaisuriya K, Dung N, Hoa PTT, Sanchaisuriya P, Fucharoen G, Fucharoen S, Schelp FP: Burden of anemia in relation to thalassemia and iron deficiency among Vietnamese pregnant women. Acta Haematol 2013;130:281-287.

5 Pasricha SR, Casey GJ, Phuc TQ, Mihrshahi S, MacGregor L, Montresor A, Tien N, Biggs BA: Baseline iron indices as predictors of hemoglobin improvement in anemic Vietnam- ese women receiving weekly iron-folic acid supplementation and deworming. Am J Trop Med Hyg 2009;81:1114-1119.

6 Modell B, Darlison M: Global epidemiology of haemoglobin disorders and derived service indicators. Bull World Health Organ 2008;86: 480-487.

7 Pasricha SR, Drakesmith H, Black J, Hipgrave D, Biggs BA: Control of iron deficiency anemia in low- and middle-income countries. Blood 2013;121:2607-2617. 